\title{
Deconstrucción del "buen profesor”. Análisis de las características formales y no formales de la docencia universitaria
}

\author{
Deconstructing the notion of a "good teacher". An analysis of the forman and non-formal \\ characteristics of university teaching
}

Casero Martínez, Antonio (Universitat de les Illes Balears)

\begin{abstract}
Resumen
Antecedentes: la evaluación de la calidad del profesorado universitario, desde el punto de vista del alumnado, se ha centrado principalmente en los aspectos más formales del ejercicio docente, desatendiendo así otros factores de tipo personal.

Objetivo: identificar y analizar las cargas de cada uno de los distintos factores que conforman la idea de la buena docencia según el alumnado. Es decir, detectar la ecuación que da lugar al esquema mental de "buen profesor" o "buena profesora”.

Método: encuesta transversal, elaborada a partir de los resultados de revisiones e investigaciones propias que permitieron conocer la identidad de los distintos factores implicados en la evaluación de la docencia por parte del alumnado. La muestra es de 885 alumnos de la Universitat de les Illes Balears, obtenidos mediante un procedimiento de muestreo mixto y polietápico.

Resultados: la solución factorial de cuatro elementos, que en orden de mayor a menor varianza explicada son: cualidades personales, competencia formal, cumplimiento, y materia. El análisis de regresión determina que cada uno de los 4 factores presenta un efecto estadísticamente significativo sobre la consideración de buen docente, donde el primero y el segundo son, en suma, responsables del 93\% de toda la varianza explicada por el modelo.

Conclusiones: el gran peso que las características no formales presentan en la ecuación resultante abre la discusión en torno a la importancia de las cualidades personales del profesorado como elementos que pueden estar modulando, más allá de la satisfacción del alumnado, el propio proceso enseñanza-aprendizaje.
\end{abstract}

Palabras clave: Evaluación de la docencia, calidad docente, satisfacción alumnado

\begin{abstract}
Background: Student appraisals of teaching quality in universities have mainly focused on more formal aspects of teaching, overlooking other personal factors.

Objective: To identify and analyse the weight of each of the different factors that contribute to the notion of what good teaching represents for students. That is, to identify the equation that constitutes a "good instructor".

Method: A crosscutting survey, based on the results of reviews and research by the author, leading to the identification of the different factors involved in student appraisals of university teaching. The sample was 885 students from the University of the Balearic Islands, obtained through multistage mixed sampling.

Results: A solution of four factors. In order of explained variance, from highest to lowest, they are personal qualities, teaching competence, compliance and subject matter. From the regression analysis that was conducted, each of the four factors was found to have a statistically significant effect on appraisals of good teaching, with the first and second factors jointly accounting for 93\% of all the model's explained variance.

Conclusions: The big impact of characteristics unrelated to teaching in the resulting equation opens up debate on the relevance of an instructor's personal qualities, not only in contributing to student satisfaction but also in influencing the teaching and learning process.
\end{abstract}

Keywords: Appraisals of teaching, teaching quality, student satisfaction.

Fecha de recepción 25-Febrero-2015

Fecha de aprobación 02-Julio-2016

Fecha de publicación 02-Julio-2016
Reception Date 2015-February 15

Approval Date 2016-July-02

Publication Date: 2016-July-02

\section{Autor de contacto / Corresponding author}

Antonio Martínez Casero. Universitat de les Illes Balears. Departamento de Pedagogía Aplicada y Psicología de la Educación. (España). a.casero@uib.es 
La evaluación de la calidad de la docencia universitaria por parte del alumnado constituye, aún hoy, uno de los puntos más polémicos del sistema de evaluación de las universidades. Con casi un siglo a sus espaldas, su aplicación sigue siendo objeto de continuo análisis psicométrico en sus adaptaciones a los cambios que proponen las nuevas propuestas didácticas (Tejedor, 2012). La mayor reticencia proviene del sector del profesorado con concepciones arraigadas sobre determinados factores que sesgarían las opiniones que los alumnos proporcionan sobre la docencia de sus profesores, ignorando la numerosa producción científica que concluye que ninguno de los supuestos factores de sesgo ha demostrado un efecto sustancial (Theall \& Feldman, 2007), o como mucho se ha detectado algún que otro débil impacto (Marsh, 2007).

\section{Garantías del constructo}

Los estudios centrados en el análisis de la evaluación docente han focalizado su interés sobre la dimensionalidad, la fiabilidad y, muy especialmente, la validez. Así, el debate entre la multidimensionalidad y la unidimensionalidad, representado originalmente por los estudios de Marsh (1987), en los que defendía que la docencia es una actividad compleja y multidimensional, encontraron respuesta en las investigaciones de Abrami y D’Apollonia (1990) y, Cashin y Downey (1992) que sin negar la existencia de la multidimensionalidad, sí cuestionaron la contundencia de Marsh al hallar, tras la revisión de los meta-análisis de Feldman (1976), ítems con pesos similares en más de una dimensión. En nuestro país las investigaciones apoyan mayoritariamente la existencia de un modelo multidimensional (Escudero, 2000). Del mismo modo, existen voces que manifiestan la tendencia de los alumnos a globalizar su respuesta en los cuestionarios (González Such, Jornet, Suárez \& Pérez Carbonell, 1999), apoyando así, también, la existencia de algo parecido a un "factor general", similar a la idea del Factor General de Inteligencia frente a la descomposición factorial que habitualmente constituyen las pruebas de inteligencia. Por su parte, Apodaca y Grad, tras un trabajo de análisis dimensional utilizando técnicas paramétricas y no paramétricas, concluyen que los resultados conducen al soslayo de polémicas improductivas, "la contraposición entre una orientación unidimensional o multidimensional se presenta innecesaria al poder contemplar el constructo tanto como unidimensional o multidimensional. En definitiva, serían los usos evaluativos los que definirían la necesidad de utilizar puntuaciones unidimensionales o multidimensionales" (Apodaca \& Grad, 2002, 406). Por otro lado, parece existir acuerdo en el contenido de las dimensiones, no así en su número. El problema es menor de lo que parece ya que aquellas propuestas con un número reducido de dimensiones utilizan macrocategorías que aparecen pormenorizadas en las propuestas con un gran número de dimensiones, siendo entre seis y nueve el número de dimensiones más habitual. Mayor en el caso de evaluaciones con orientación formativa, y menor cuando la orientación es sumativa o de acreditación, distinguiendo así la orientación formativa cómo única evaluación conceptualmente adecuada (Tejedor \& Jornet, 2008).

Respecto a la fiabilidad de los cuestionarios de opinión. Ésta aparece como altamente satisfactoria (Aparicio, Tejedor \& Sanmartín, 1982), tanto a nivel de consistencia interna de los elementos que componen los instrumentos -excepto con grupos muy pequeños-, como de estabilidad de las respuestas en el tiempo, siendo este último aspecto un indicador de validez.

La validez ha sido el aspecto más discutido y estudiado. Las investigaciones muestran sus mejores resultados en los análisis estructurales paramétricos, y mejores aún en los no paramétricos (Apodaca \& Grad, 2002) realizados sobre instrumentos multidimensionales. Los resultados del análisis convergente-discriminante resultan entre moderadamente satisfactorios (García Ramos, 1999), y satisfactorios (Marsh \& Hocevar, 1984), al igual que ocurre con los estudios de 
validez multisección, aún presentando complicaciones metodológicas (Garcia Garduño, 2000). Otro apoyo a la validez lo constituyen las satisfactorias aplicaciones transculturales (Marsh, Hau, Chung \& Siu, 1997).

Por último, las correlaciones obtenidas entre las valoraciones de los alumnos y las valoraciones de los ex alumnos respaldan la validez del constructo (Seldin, 1993). También se ha observado una relación significativa con las autoevaluaciones del profesorado, alcanzando en este caso un nivel moderadamente aceptable (Drews, Burroughs \& Nokovick, 1987). Los estudios que relacionan las evaluaciones del alumnado con las evaluaciones realizadas por colegas no dan soporte a la validez, poniendo en duda la capacidad de los colegas para evaluar el constructo (Aparicio, Tejedor \& Sanmartín, 1982; De Miquel, 1991). Un tipo de investigación que ha dado un fuerte respaldo a la validez es la realizada a partir de las evaluaciones por observadores externos entrenados-, especialmente las centradas en la claridad docente. Éste parece ser el aspecto que nuclea el constructo de calidad docente (Hines, Cruickshank \& Kennedy, 1985).

\section{Pero, ¿cómo es un buen profesor?}

La indefinición del constructo ha sido otro aspecto muy aludido (Álvarez, García Jiménez \& Gil, 1999; García Ramos, 1999; Pascual \& Gaviria, 2004). La investigación se ha venido centrando en el intento por detectar qué elementos son los que conforman el trabajo docente "de calidad", certificación imprescindible en cualquier profesión que se precie. Los autores utilizan de forma muy similar términos como tarea docente, excelencia docente, eficacia docente, buen profesor, actuación docente, docencia de calidad, competencia docente, o función docente, siendo aproximadamente igual en número cuando sustituimos docente por enseñanza. El procedimiento metodológico utilizado para deducir cuales son las características que conforman el constructo objeto de estudio ha sido también un asunto de relevante estudio. En este sentido, los procedimientos han ido desde la reflexión teórica de lo que debe ser un buen profesor hasta investigaciones empíricas de corte cualitativo ahondando en los esquemas mentales del alumnado.

Según Ericksen (1985) un buen profesor: selecciona y organiza el material del curso, guía a los estudiantes para el registro e integración de la información de modo que puedan recordarlo fácilmente, es competente en los procedimientos y métodos de su disciplina, mantiene la curiosidad intelectual de sus alumnos, y promueve el aprendizaje independiente.

Un buen profesor, según Elton (1987) es, o está: bien organizado, bien preparado, interesado en la materia, amigable, flexible, servicial, creativo, entusiasta, claro, interesado por los estudiantes, abierto, sistemático, comprometido, y entregado.

Para De la Cruz (1999), el profesor universitario debe ser un especialista al más alto nivel de su área de conocimiento. Esta especialización tiene que estar vinculada a la investigación sobre el contenido de su materia y la docencia de la misma. Formado profesionalmente de manera sistematizada en cada una de las tareas que tiene que desempeñar, principalmente: investigación, docencia y gestión. Motivado para la investigación y la docencia de su asignatura. Con entusiasmo, interés y vocación por ella. Poseer ciertos rasgos de personalidad. Entre ellos: paciencia, tolerancia, apertura, adaptación, flexibilidad y sentido del humor. Poseer ciertas habilidades personales básicas. Entre éstas: facilidad para las relaciones interpersonales, habilidades para la comunicación, control del estrés, tolerancia a la frustración, etc. Con habilidades docentes específicas: organización y estructuración de los conocimientos a impartir, planificación a largo y corto plazo de las actividades docentes, claridad expositiva, presentación de los contenidos de manera que despierte el interés de los estudiantes y promueva el aprendizaje independiente en los alumnos, organizador y gestor de situaciones y recursos de 
aprendizaje. Poseer una actitud crítica y reflexiva con respecto a su propia actuación como profesor. Innovador, abierto al cambio.

El profesor profesional, según Ramsden (1992), posee un amplio repertorio de habilidades docentes específicas, no olvida que sus metas son el aprendizaje de los estudiantes, escucha y aprende de sus alumnos, evalúa constantemente su actuación docente, piensa que enseñar es hacer posible el aprendizaje, enseña con entusiasmo, muestra preocupación y respeto por los alumnos, tiene facilidad para hacerse entender por los estudiantes, hace del estudiante un aprendiz autónomo, Usa métodos que exigen al estudiante aprender activa y cooperativamente, da feedback de alta calidad a sus estudiantes sobre sus trabajos, enseña los conceptos claves de su materia y evita sobrecarga de trabajo.

La enseñanza eficaz, según Brown (1993), debe tener las siguientes características: conocimiento en profundidad de la asignatura, comunicación fluida con los alumnos, conocimiento de los estilos de aprendizaje del alumno, conocimiento de la didáctica universitaria.

Las características del buen profesor, como conclusión de estudios sobre los docentes que reciben las mejores calificaciones en sus evaluaciones son las siguientes (Gros y Romaña, 2004): debe saber de su materia, preparar bien las clases, explicar con claridad y orden, escribir en la pizarra con claridad, orden y buena letra, ser capaz de realizar un trabajo en equipo, poseer cierta sensibilización por su entorno social y cultural, ser capaz de ponerse en el lugar del alumno, resultar asequible a los estudiantes, ser algo teatral, hacer participar a los estudiantes, llegar a clase puntualmente, ser educado, simpático, y tener buena presencia física.

Criterios que establecen algunas universidades para otorgar la excelencia docente (Dunkin \& Precians, 1992): interés y entusiasmo al enseñar $y$ promover el aprendizaje, interés en promover la mejora de la enseñanza través de innovaciones, dominio de la materia y actualización en su campo de estudio, captación de la necesidad de los estudiantes, toma en cuenta el feedback de sus colegas y alumnos sobre su enseñanza, habilidad para organizar los materiales de la clase y presentarlos de manera estimulante, habilidad para evaluar y dar feedback útil a sus estudiantes, habilidad para estimular la curiosidad y el aprendizaje independiente en los estudiantes principiantes y trabajo creativo que los estudiantes de cursos superiores e, interés y compromiso en promover la excelencia en enseñanza entre sus colegas.

La Agencia Nacional de Evaluación de la Calidad y Acreditación española, a través de su programa DOCENTIA, establece las dimensiones para la evaluación del profesorado: Planificación, Acción, Comprobación, y revisión. De ellas se derivan 10 subdimensiones que atañen, y operativizan las 4 anteriores: programación docente, actividades de organización y coordinación docente, consecución del encargo docente, fortalecimiento de asignaturas, ampliación de tareas, consecución de objetivos formativos y satisfacción de los estudiantes, formación recibida, innovación-proyectos, innovaciónmateriales e, innovación-reconocimiento.

El esquema mental del alumnado sobre el buen profesor, como resultado de investigaciones cualitativas, recogido en la figura 1, cuyo objetivo es desmenuzar la idea que ha conformado el alumnado en relación al constructo en cuestión (Casero, 2010a). 
Casero-Martínez, Antonio (2016). Deconstrucción del "buen profesor”. Análisis de las características formales y no formales de la docencia universitaria. RELIEVE, 22(2), art. 4. doi: http://dx.doi.org/10.7203/relieve.22.2.9419

FIGURA 1. Esquema cognitivo del alumnado sobre la idea de buen docente

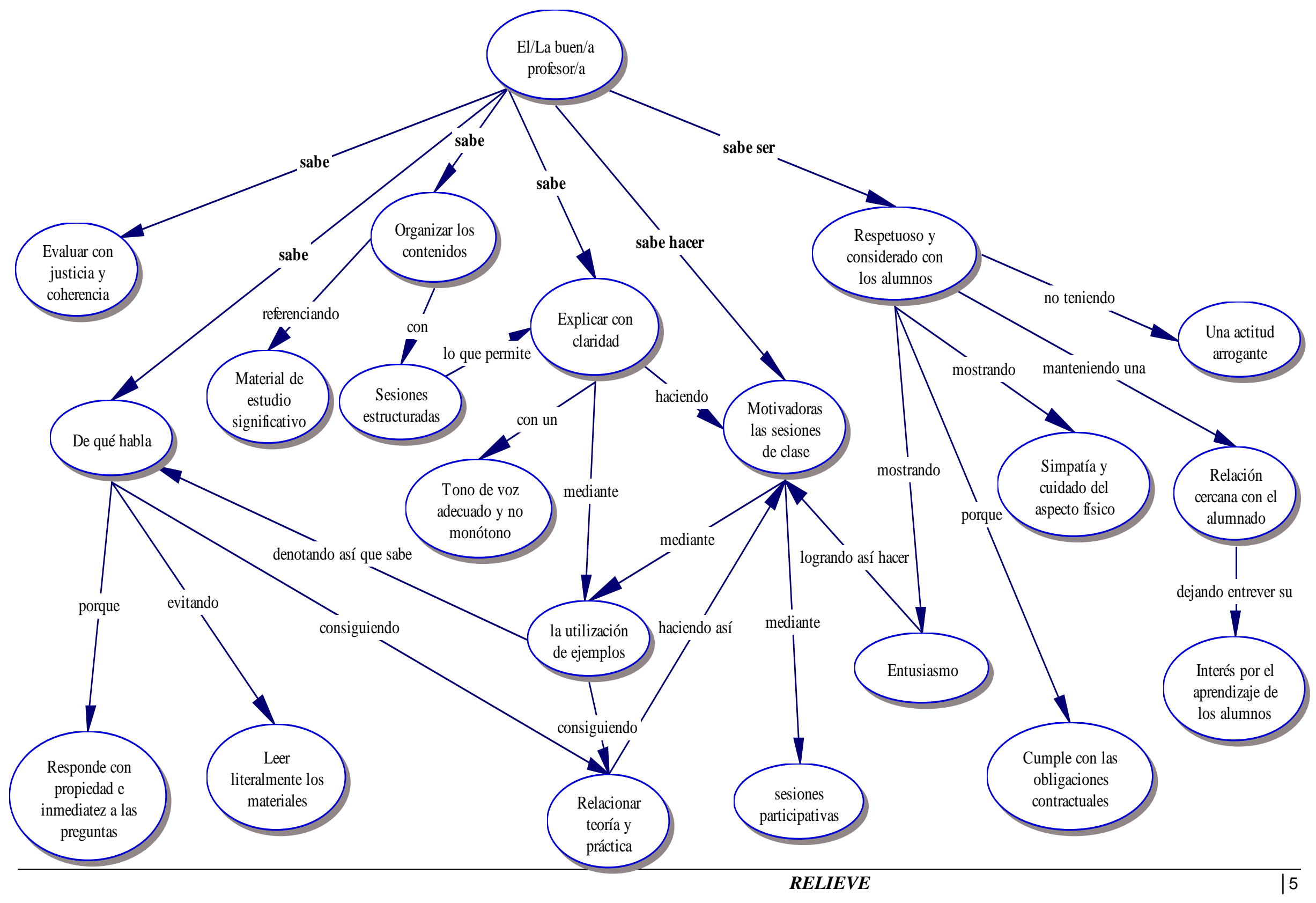


Son muchos los puntos en común que presentan las distintas concepciones de la buena docencia, y no todas hacen referencia, de manera estricta, a elementos formales, académicos o profesionales del docente. Han aparecido diversos elementos de carácter personal, variables que más tienen que ver con la forma de ser y sus habilidades como persona que con su formación o grado de dominio, y que seguro algún impacto sobre el constructo deben tener. Así, el presente trabajo pretende detectar qué peso tiene cada uno de los distintos factores que conforman la idea de la buena docencia según el alumnado. Es decir, obtener la ecuación que da lugar al esquema mental de "buen profesor" o "buena profesora”, partiendo de los resultados de revisiones e investigaciones que han permitido conocer la identidad de los distintos factores implicados en la evaluación de la docencia por parte del alumnado.

\section{Método}

La estrategia metodológica utilizada para alcanzar el objetivo ha sido la encuesta transversal, aplicada a través de un cuestionario compuesto de un total de 30 ítems, donde el alumnado debe expresar en una escala de 0 a 10 el grado de acuerdo con cada una de las 30 afirmaciones. Los 20 ítems primeros, del A1 al A20, son el producto de un estudio de Casero (2008), donde alumnos y profesores expresaron el grado de importancia de cada uno de los 92 elementos recogidos como inventario del conjunto de ítems utilizados en las universidades españolas, aplicado tanto al alumnado como al profesorado. El resultado fue una solución obtenida tras un proceso de análisis mixto en el que se conjugó el uso de indicadores robustos con análisis factorial exploratorio y análisis de contenido. Los 10 ítems restantes, del B1 al B10, son el resultado de una investigación cualitativa realizada por el mismo autor en la que se abordaban los aspectos no formales de la percepción de la calidad docente detectados mediante, en una primera fase, cuestionarios cualitativos dirigidos a alumnado, y grupos de discusión en la fase final (Casero, 2010a). En la tabla 3 se exponen todos los elementos que configuran el instrumento.

La muestra se compone de 885 alumnos (284 hombres y 601 mujeres; media de edad de 21.32 años y una desviación estándar de 4.17) de la Universitat de les Illes Balears, obtenidos mediante un procedimiento de muestreo mixto y polietápico, con selección de las unidades primarias -profesores- mediante muestreo aleatorio simple, de las unidades secundarias -asignaturas- mediante asignación del profesor, y de las unidades finales alumnos- mediante muestreo incidental en el aula, resultando un error muestral para el conjunto de la muestra del $3.82 \%$, estimado para un nivel de confianza del $95 \%$ y bajo la condición más desfavorable de $\mathrm{p}=\mathrm{q}=.5$.

\section{Resultados}

El análisis de las respuestas lo hemos dividido en tres partes. En primer lugar, aquel que hace referencia a las garantías métricas del instrumento utilizado. En segundo lugar se ha realizado el análisis que permite detectar la presencia de los factores que conforman el constructo objeto de estudio. Finalmente, el análisis de estos factores nos ha permitido presentar la ecuación que resume el modelo general de la valoración de la calidad docente según el alumnado.

\section{Garantías métricas}

El análisis de la fiabilidad por consistencia interna arroja un valor alfa de Cronbach basado en los elementos estandarizados de 0.96 , valor más que satisfactorio. Los valores alpha eliminando el ítem de los 30 elementos que componen la escala utilizada han oscilado entre 0.95 y 0.96 .

Como estimación de la validez relativa al criterio hemos realizado un análisis de regresión múltiple donde las variables independientes han sido 29 ítems, todos menos el A20, asumiendo éste el papel de variable dependiente al resumir la valoración global del docente. El resultado obtenido -ver tabla 1nos informa de la alta capacidad predictiva que 
el conjunto de los 29 ítems tienen sobre la valoración global del docente, siendo ésta del
$81 \%$, valor que confiere altas garantías de validez concurrente a la medida del constructo.

Tabla 1. Correlación múltiple

\begin{tabular}{ccccc}
\hline Modelo & $\mathrm{R}$ & $\begin{array}{c}\mathrm{R} \\
\text { cuadrado }\end{array}$ & $\begin{array}{c}\text { R cuadrado } \\
\text { corregida }\end{array}$ & $\begin{array}{c}\text { Error estándar } \\
\text { de la } \\
\text { estimación }\end{array}$ \\
\hline 1 & .91 & .82 & .81 & .98 \\
\hline
\end{tabular}

\section{Estructura factorial}

La tabla 2 recoge los resultados que evalúan la pertinencia de aplicar un análisis factorial a nuestros datos. El índice de KMO arroja un resultado, que según el baremo de Kaiser, es muy bueno para el índice obtenido. Por su lado, la prueba de esfericidad nos informa del cumplimiento del requisito de alta correlación entre las variables. Estos resultados son altamente satisfactorios, lo que legitima la realización del análisis factorial.

Tabla 2. Pruebas KMO y de esfericidad

\begin{tabular}{ccc}
\hline \multirow{2}{*}{$\begin{array}{c}\text { Medida de adecuación } \\
\text { muestral de } \\
\text { Kaiser-Meyer-Olkin }\end{array}$} & \multicolumn{2}{c}{ Prueba de esfericidad de Bartlett } \\
\cline { 2 - 3 } & $\begin{array}{c}\text { Ji-cuadrado } \\
\text { aproximado }\end{array}$ & Significación \\
\hline 0.97 & 17434.23 & $<.01$ \\
\hline
\end{tabular}

El análisis factorial exploratorio ha sido aplicado mediante el método de extracción de análisis de componentes principales, el más utilizado en este tipo de trabajos. Sobre esta solución se ha realizado una rotación mediante el método de normalización Varimax con Kaiser. Este método de rotación ortogonal es el que minimiza el número de variables que tienen saturaciones altas en cada factor, simplificando la interpretación de los mismos. Así, el análisis arroja una solución de 4 factores que explican el $64.73 \%$ de la varianza total, donde el primero es responsable del $25.48 \%$ de la varianza total, el segundo explica el $23.95 \%$, siendo $10.01 \%$ y $5.22 \%$ los porcentajes explicados por el tercer y cuarto factor, respectivamente.

En la tabla 3 se presenta la correlación de cada elemento con el factor que mantiene una mayor saturación. A partir de los contenidos de los elementos asociados a los factores hemos denominado a los mismos como se indica a continuación:
Factor I: Cualidades personales, cuyos contenidos versan sobre las características personales extraprofesionales del docente, razón por la que recibe esta denominación.

Factor II: Competencia docente, porque el contenido de los ítems versa sobre la capacidad para motivar, despertar interés, trasmitir el conocimiento, claridad en la explicación, saber responder de forma precisa a las dudas, y relacionar conceptos y contenidos, principalmente. Este conjunto de comportamientos evocan dominio de los contenidos y habilidades para trasmitirlos de forma estimulante. A nuestro entender este es el núcleo, en sentido estricto, de la competencia docente.

Factor III: Cumplimiento formal, en relación al cumplimiento de las obligaciones contractuales del docente en el trato con el alumnado.

Factor IV: Materia, por verse saturado únicamente por los aspectos propios de la 
materia impartida, con aparente independencia de las variables propias del docente.

Tabla 3. Correlaciones entre ítems y factores

\begin{tabular}{|c|c|c|c|c|}
\hline Ítem & $\mathrm{I}$ & II & III & IV \\
\hline $\begin{array}{l}\text { A1. Da a conocer el programa (objetivos, contenidos, metodología, evaluación, } \\
\text { extensión, desarrollo), a principio de curso }\end{array}$ & & .55 & .54 & \\
\hline $\begin{array}{l}\text { A2. El programa se desarrolla a un ritmo que permite tratar de forma adecuada y } \\
\text { rigurosa todos los temas }\end{array}$ & & .69 & & \\
\hline A3. Lo explicado en clase responde al programa de la asignatura & & .66 & & \\
\hline A4. Es accesible durante el horario de tutorías & & & .72 & \\
\hline A5. Cumple con sus obligaciones de atención a los estudiantes & & & .66 & \\
\hline A6. Las clases están bien preparadas, organizadas y estructuradas & & .76 & & \\
\hline A7. Explica con claridad los conceptos implicados en cada tema & & .79 & & \\
\hline $\begin{array}{l}\text { A8. Cuando introduce conceptos nuevos los relaciona, si es posible, con los ya } \\
\text { conocidos }\end{array}$ & & .73 & & \\
\hline $\begin{array}{l}\text { A9. Responde puntualmente y con precisión a las cuestiones que le planteamos en } \\
\text { clase sobre conceptos de la asignatura u otras cuestiones }\end{array}$ & & .65 & & \\
\hline A10. Sabe transmitir su conocimientos & & .75 & & \\
\hline $\begin{array}{l}\text { A11. Relaciona los contenidos de esta asignatura con los de otras asignaturas, } \\
\text { evitando solapamientos }\end{array}$ & & .57 & & \\
\hline $\begin{array}{l}\text { A12. La comunicación profesor/a-estudiante es fluida y espontánea, creando un } \\
\text { clima de confianza }\end{array}$ & .70 & & & \\
\hline A13. Consigue que estemos motivados/as e interesados/as por la materia & .63 & .63 & & \\
\hline A14. Se preocupa de los problemas de aprendizaje de sus alumnos/as & .66 & & & \\
\hline A15. Procura hacer interesante la asignatura & .66 & .60 & & \\
\hline A16. Es accesible y está dispuesto/a a ayudarnos & .70 & & & \\
\hline A17. Es respetuoso/a con los estudiantes & & & .59 & \\
\hline A18. Los materiales de estudio (textos, apuntes, etc.) son adecuados & & .59 & & \\
\hline $\begin{array}{l}\text { A19. Las clases prácticas son un buen complemento de los contenidos teóricos de la } \\
\text { asignatura }\end{array}$ & & .53 & & \\
\hline B1. Este/a profesor/a tiene gran sentido del humor & .76 & & & \\
\hline B2. El/La profesor/a tiene muy buena reputación & .65 & & & \\
\hline B3. Este/a profesor/a me cae bien & .79 & & & \\
\hline B4. Este/a profesor/a me parece una persona modesta & .66 & & & \\
\hline B5. Es muy simpático/a & .82 & & & \\
\hline B6. Este/a profesor/a me resulta atractivo/a & .51 & & & \\
\hline B7. Se nota que disfruta dando clase & .61 & & & \\
\hline $\begin{array}{l}\text { B8. Para los créditos que tiene esta asignatura, creo que se nos ha sobrecargado de } \\
\text { trabajo (trabajos, lecturas...) }\end{array}$ & & & & .79 \\
\hline B9. Esta asignatura tiene fama de ser una asignatura "hueso" & & & & .75 \\
\hline B10. Esta asignatura es muy importante para el conjunto de mis estudios & & & & .42 \\
\hline
\end{tabular}


Modelo general de la valoración de la docencia

A partir de los resultados obtenidos mediante el análisis factorial se ha procedido al análisis de regresión múltiple de la valoración global de la calidad del docente ítem A20-, siendo las puntuaciones factoriales -obtenidas mediante el método de regresiónde los 4 factores detectados las variables predictoras. El método utilizado ha sido el de pasos sucesivos -stepwise-, que permite la construcción de la ecuación lineal múltiple mediante la selección de variables paso a paso, que frente a otros métodos, presenta la ventaja de admitir que una variable seleccionada en un paso puede ser eliminada en otro posterior. El procedimiento de inclusión y exclusión de variables se basa en calcular un valor $F$ para cada variable predictora, y se incluyen en la ecuación de regresión las variables cuya probabilidad de F sea menor que $0.05 \mathrm{y}$ se excluyen las variables cuya probabilidad de $\mathrm{F}$ sea mayor que 0.10 .

Tabla 4. Resumen del modelo de regresión lineal múltiple sobre la valoración global del docente

\begin{tabular}{|c|c|c|c|c|c|c|c|c|c|}
\hline \multirow[b]{2}{*}{ Modelo* } & \multirow[b]{2}{*}{$\mathrm{R}$} & \multirow[b]{2}{*}{$\mathrm{R}^{2}$} & \multirow[b]{2}{*}{$\begin{array}{c}\mathrm{R}^{2} \\
\text { corregida }\end{array}$} & \multirow[b]{2}{*}{$\begin{array}{c}\text { Error } \\
\text { estándar }\end{array}$} & \multicolumn{5}{|c|}{ Estadísticos de cambio } \\
\hline & & & & & $\begin{array}{c}\text { Cambio } \\
\text { en R } \\
\text { cuadrado }\end{array}$ & $\begin{array}{c}\text { Cambio } \\
\text { en F }\end{array}$ & gl1 & gl2 & $\begin{array}{c}\text { Significación } \\
\text { del cambio } \\
\text { en F }\end{array}$ \\
\hline 1 & .64 & .40 & .40 & 1.75 & .40 & 598.62 & 1 & 883 & $<.01$ \\
\hline 2 & .85 & .73 & .73 & 1.18 & .33 & 1064.86 & 1 & 882 & $<.01$ \\
\hline 3 & .89 & .78 & .78 & 1.06 & .05 & 216.59 & 1 & 881 & $<.01$ \\
\hline 4 & .89 & .79 & .79 & 1.05 & $<.01$ & 10.19 & 1 & 880 & $<.01$ \\
\hline
\end{tabular}

Modelo 1. Variables predictoras: (Constante), Competencia formal

Modelo 2. Variables predictoras: (Constante), Competencia formal, Cualidades personales

Modelo 3. Variables predictoras: (Constante), Competencia formal, Cualidades personales, Cumplimiento

Modelo 4. Variables predictoras: (Constante), Competencia formal, Cualidades personales, Cumplimiento, Materia Variable dependiente: Ítem A20 Globalmente pienso que es un buen/a profesor/a

El proceso por pasos, expuesto en la tabla 4, presenta una solución en 4 pasos. El resultado de la correlación múltiple al cuadrado corregida indica que las variables seleccionadas en el modelo permiten predecir el 79\% de la dimensión global.

Tabla 5. Análisis de la varianza del modelo de regresión

\begin{tabular}{lccccc}
\hline $\begin{array}{c}\text { Fuentes de } \\
\text { variación }\end{array}$ & $\begin{array}{c}\text { Suma de } \\
\text { cuadrados }\end{array}$ & g.l. & Media cuadrática & F & Significación \\
\hline Regresión & 3576.47 & 4 & 894.12 & 806.90 & $<.01$ \\
Residual & 975.12 & 880 & 1.11 & & \\
Total & 4551.58 & 884 & & & \\
\hline
\end{tabular}

El análisis de la varianza del modelo de regresión presenta significación estadística, indicando que la pendiente del plano de regresión es distinta de cero; es decir, existe ajuste lineal entre el conjunto de variables 
predictoras y la variable dependiente -ver tabla 5-.

Respecto a las garantías del modelo, el hecho de que el análisis de la varianza ofrezca un resultado significativo y que existan coeficientes de regresión también significativos -ver tabla 6-, permite despejar la presencia de colinealidad. Esta ausencia se ve confirmada por los valores de tolerancia obtenidos, cuyos valores se encuentran entre .99 y 1.
Para poder valorar los pesos relativos hemos utilizado los coeficientes Beta, éstos indican la cantidad de cambio, en puntuaciones estándar, que se producirá en la variable dependiente por cada cambio de una unidad en la correspondiente variable predictora, manteniendo constantes el resto de variables predictoras. Así, este coeficiente es el que permite comparar directamente la importancia de cada variable predictora, con independencia a la escala en que esté medida.

Tabla 6. Coeficientes de regresión

\begin{tabular}{lccccc}
\hline & \multicolumn{2}{c}{$\begin{array}{c}\text { Coeficientes no } \\
\text { estandarizados }\end{array}$} & $\begin{array}{c}\text { Coeficientes } \\
\text { estandarizados }\end{array}$ & & \\
\cline { 2 - 4 } \multicolumn{1}{c}{ Variables predictoras } & $\mathrm{B}$ & $\begin{array}{c}\text { Error } \\
\text { estándar }\end{array}$ & Beta & $\mathrm{t}$ & Signif. \\
\hline (Constante) & 7.40 & .04 & & 209.00 & $<.01$ \\
Competencia docente & 1.44 & .04 & .64 & 40.74 & $<.01$ \\
Cualidades personales & 1.30 & .04 & .57 & 36.59 & $<.01$ \\
Cumplimiento & .52 & .04 & .23 & 14.80 & $<.01$ \\
Materia & .11 & .04 & .05 & 3.19 & $<.01$ \\
\hline
\end{tabular}

Los coeficientes estandarizados permiten observar la importancia relativa de cada factor, donde la competencia docente aparece es el factor más importante como predictor de la valoración global del docente, seguido muy de cerca por las cualidades personales, con algo menos de la mitad de importancia aparece el cumplimiento y en último lugar, y lejos del factor anterior, se encuentra la materia.

\section{Discusión}

Los resultados obtenidos han permitido presentar, en primer lugar, un instrumento de investigación fiable, con alta consistencia interna, y válido, con valores similares a los obtenidos por Muñiz, García y Virgos (1991) y los presentados en el Informe General sobre la Calidad Docente del Profesorado de la Universidad de La Laguna (2003), superando incluso los hallazgos de Mateo y Fernández (1993), Mairata y Servera (1996), Capelleras y Veciana (2001), y Muñoz, Ríos y Abalde
(2002), confiriendo alta garantía a la validez concurrente del constructo.

Por otro lado, y como principal contribución del trabajo al haber alcanzado el objetivo propuesto, se han descubierto y presentado los pesos relativos con los que cada uno de los grandes factores determinan la puntuación global de la valoración del docente por parte del alumnado. Así, los resultados arrojan la siguiente ecuación: valoración global del docente $=$ Competencia docente (.66) + Cualidades personales (.55) + Cumplimiento (.24) + Materia (.04). Estos hallazgos confirman el enfoque multidimensional de la calidad docente defendido por muchos autores, entre los que destaca Marsh (1987). A la vez, también se confirma la presencia de un factor general, observado por el alto poder predictivo que los elementos específicos tienen sobre la valoración global de la docencia. Este resultado es totalmente coherente con los obtenidos por Apodaca y Grad (2002), cuando señalan que el constructo puede ser observado 
tanto desde una perspectiva unidimensional como multidimensional, siendo la finalidad de la evaluación la que definirá cómo utilizar los resultados -sumativa y/o formativa-.

La “deconstrucción” del constructo permite observar la presencia de dos grandes entidades, la formal y la no formal, con pesos demasiado similares para descuidar la atención sobre la importancia de la segunda. Es obvio que las variables "extraprofesionales" procedentes del propio docente han jugado un papel central en este trabajo. Su inclusión respondió a la creciente sospecha de su influencia tras conversaciones informales con alumnos y profesores, y el apoyo parcial de la revisión de la literatura y de investigaciones propias. Es por ello que la presencia de esta variable en los resultados no ha causado sorpresa. Lo inimaginable ha sido el valor recibido: un $83 \%$ de la capacidad de cambio exhibida por la dimensión competencia docente. Concretando, "ser simpático/a" y "caer bien”, como elementos que mantienen la correlación más alta con el factor cualidades personales, son los que mejor reflejan la dimensión personal para el alumnado, con importante impacto sobre la valoración global del docente. Por su parte, el elemento que mejor representa al factor competencia docente ha sido el que hace referencia a la claridad expositiva. Este último resultado es congruente con la literatura científica revisada, Hines, Cruickshank y Kennedy (1985), y Apodaca y Grad (2002), al situar la claridad en la exposición como núcleo del constructo aquí denominado calidad docente.

Parece que el manejo de impresiones por parte del profesor respecto al alumno podría explicar el efecto hallado, por lo menos en parte, aunque, claro está, bajo la suposición de la existencia de un comportamiento proactivo del profesor dirigido a generar una impresión favorable en el alumno; idea que entronca con los hallazgos del paradigma del Dr. Fox, donde la denominada "seducción académica" ha relatado efectos sustanciales sobre las valoraciones de los estudiantes, aunque no tanto como lo aquí observado, hecho que sugiere la existencia de, cuanto menos, alguna otra razón que lo explique (Casero, 2010b). La discusión nos lleva, entonces, a la otra parte: el alumno. La población se caracteriza por sujetos de ambos sexos, con edades comprendidas, mayoritariamente, entre $18 \mathrm{y}$ 25 años, recién salidos del estadio adolescente, y expuestos diariamente a figuras de poder que decidirán sobre la conveniencia de su continuidad en el sistema de educación superior. Bajo estas circunstancias resulta lícito pensar que la presencia de "afinidad" entre las características personales del docente y las del alumno genere un "efecto halo" que repercuta sobre la valoración global del docente.

Por último, otro aspecto a discutir es la influencia que la percepción favorable, por parte del alumno, de las características personales del docente puede tener sobre la propia relación entre ambos (Spilt, Koomen \& Thijs, 2011), sobre la construcción de las mutuas expectativas (Rubie-Davies, 2010), y sobre el proceso de enseñanza-aprendizaje. Presumiblemente, la mencionada "afinidad" se perfila como un elemento facilitador de dicho proceso, favoreciendo la predisposición del alumno, y llegando a constituir un elemento motivador a tenor de las declaraciones emitidas por los propios alumnos. Este planteamiento ocupa una posición central en el contexto empresarial, donde poco venden los comerciales que exponen el producto con apatía y poca convicción. Con ello no queremos incurrir en el error de proponer un modelo docente sobre la base del modelo empresarial. Esta asunción desvirtuaría, en esencia, la finalidad de la educación. Sin embargo, creemos oportuno concluir que las características personales deseables en cualquier individuo juegan, en el caso del docente, un papel sustancial en el proceso educativo. Más allá de representar un sesgo sobre su adecuado ejercicio docente, constituye el elemento "ideal" que, junto con las competencias "formales", conforma el perfil de un buen docente. 


\section{Referencias}

Abrami, P.C. \& D’Apollonia, S. (1990). The dimensionality of ratings and their use in personnel decisions. En M. Theall y J. Franklin (Comps.), Student ratings of instruction: Issues for improving practice. New directions for teaching and learning, (pp. 97-111). San Francisco: Jossey-Bass.

Agencia Nacional de Evaluación de la Calidad y Acreditación. Consultado 24-7-2015. http://www.aneca.es

Álvarez, V., García Jiménez, E. \& Gil, J. (1999b). Características de la docencia mejor evaluada por los alumnos en las diferentes áreas de enseñanza universitaria. Revista Española de Pedagogía, 214, 445-464

Aparicio, J.J., Tejedor, F.J. \& Sanmartín, R. (1982). La enseñanza universitaria vista por sus alumnos: Un estudio para la evaluación de los cursos de la enseñanza superior. Madrid: ICE/ Universidad Autónoma de Madrid.

Apodaca, P. \& Grad, H. (2002). Análisis dimensional de las opiniones de los alumnos universitarios sobre sus profesores: Comparación entre técnicas paramétricas y no-paramétricas. Revista de Investigación Educativa, 20(2), 385-409.

Brown, G. (1993). Staff Development in Britain. Restrospective and prospective. Looking back and looking forward. En L.M. Lázaro Lorente (Ed.), Formación Pedagógica del Profesorado Universitario y Calidad de la Educación. Valencia: Servei de Formació Permanent, Universidad de Valencia/ CIDE.

Capelleras, J.L. \& Veciana, J.M. (2001, septiembre). Calidad de servicio en la enseñanza universitaria: Desarrollo y validación de una escala de medida. Actas del XI Congreso Nacional de ACEDE, La nueva economía: retos y oportunidades para la gestión empresarial. Zaragoza.

Casero, A. (2008). Propuesta de un cuestionario de evaluación de la calidad docente universitaria consensuado entre alumnos y profesores. Revista de Investigación Educativa, 26, 25-44.

Casero, A. (2010a). ¿Cómo es el buen profesor universitario según el alumnado? Revista Española de Pedagogía, 246, 223-242.

Casero, A. (2010b). Factores moduladores de la percepción de la calidad docente. RELIEVE, 16

http://dx.doi.org/10.7203/relieve.16.2.4135

Cashin, W.E. \& Downey, R.G. (1992). Using global student rating items for summative evaluation. Journal of Educational Psychology, 84, 563-572.

De la Cruz, M.A. (1999). Modelo de profesor y modelo de formación. En T. Hornilla (Coor.), Formación del profesorado universitario y calidad de la enseñanza. Leioa: Servicio de Publicaciones de la Universidad del País Vasco.

De Miguel, M. (Dir.). (1991). Criterios para la evaluación del profesorado. Oviedo: Departamento de Ciencias de la Educación de la Universidad de Oviedo.

Drews, D.R., Burroughs, W.J. y Nokovich, D.A. (1987). Teacher self-ratings as validity criterion for student evaluations. Teaching of Psychology, 14(1), 23-25.

Dunkin, M.J. y Precians, R.P. (1992). Awardwinning university teachers' concepts of teaching. Higher Education, 24, 483-502.

Elton, L. (1987). Teaching in higher education: appraisal and training. London: Kogan Page.

Ericksen, S.C. (1985). The essence of godd teaching. Helping students to learn and remember what they learn. San Francisco: Jossey-Bass Publishers.

Escudero, T. (2000). La voz de los estudiantes: un delicado instrumento de evaluación. Cuadernos IRC, 5, 31-38

Feldman, K.A. (1976). The superior college teacher from the students's view. Research in Higher Education, 5, 243-288.

García Garduño, J.M. (2000). Las dimensiones de la efectividad docente, validez $\mathrm{y}$ 
confiabilidad de los cuestionarios de evaluación de la docencia: síntesis de investigación internacional. En M. Rueda y F. Díaz (Comps.), Evaluación de la docencia: perspectivas actuales (pp.41-62). México DF: Paidós.

García Ramos. J.M. (1999). Análisis multirrasgo-multimétodo en la validación de instrumentos para la evaluación de la calidad docente en instituciones universitarias. Revista Española de Pedagogía, 214, 417444.

González Such, J., Jornet, J.M., Suárez, J.M. \& Pérez Carbonell, A. (1999). Análisis de tipologías de calidad docente a partir de un cuestionario de evaluación del profesorado universitario. Bordón, 51(1), 95-113.

Gros, B. \& Romañá, T. (2004). Ser Profesor. Palabras sobre la docencia universitaria. Barcelona: Octaedro.

Hines, C.V., Cruickshank, D. \& Kennedy, J.J. (1985). Teacher clarity and its relationship to student achievement and satisfaction. American Educational Research Journal, 22(1), 87-99.

Mairata, M.J. \& Servera, M. (1996). L'avaluació de la qualitat docent a la Universitat de les Illes Balears. L'Arc, 2, 4548.

Marsh, H.W. (1987). Students' Evaluation of University Teaching: Research Findings, Methodological Issues, and Directions for Future Research. International Journal of Educational Research, 11(3), 255-388.

Marsh, H.W. (2007). Students' Evaluations of University Teaching: Dimensionality, Reliability, Validity, Potential Biases and Usefulness. En R.P. Perry \& J.C. Smart (eds.), The Scholarship of Teaching and Learning in Higher Education: An EvidenceBased Perspective, (pp. 319-383). Netherlands: Springer.

Marsh, H.W. \& Hocevar, D. (1984). The factorial invariance of student evaluations of college teaching. American Educational Research Journal, 21(2), 341-366.
Marsh, H.W., Hau, K., Chung, C. y Siu, T. (1997). Students' evaluation of university teaching: Chinese version of the students' evaluations of educational quality instrument. Journal of Educational Psychology, 89, 568572.

Mateo, M.A. \& Fernández, J. (1993). Dimensiones de la calidad de la enseñanza universitaria. Psicothema, 5(2), 265-275.

Muñiz, J., García, A. \& Virgos, J.M. (1991). Escala de la Universidad de Oviedo para la evaluación del profesorado. Psicothema, 3(2), 269-281.

Muñoz, J.M., Ríos, M.P. \& Abalde, E. (2002). Evaluación docente vs. Evaluación de la calidad. RELIEVE, 8(2), 103-134. Doi: http://dx.doi.org/10.7203/relieve.8.2.4362

Pascual, I. \& Gaviria, J. L. (2004). El problema de la fiabilidad en la evaluación de la eficacia docente en la universidad: una alternativa metodológica. Revista Española de Pedagogía, 62, 229, 359-375.

Ramsden, P. (1992). Learning to teach in higher education. London: Routledge.

Rubie-Davies, C.M. (2010). Teacher expectations and perceptions of student attributes: Is there a relationship? British Journal of Educational Psychology, 80(1), 121-135.

Seldin, P. (1993). The use and abuse of student ratings of professors. The Chronicle of Her Education, p. 440.

Spilt, J.L., Koomen, H.M.Y. \& Thijs, J.T. (2011). Teacher Wellbeing: The Importance of Teacher-Student Relationships. Educational Psychology Review, 23(4), 457477.

Tejedor, F. J. (2012). Evaluación del desempeño docente. Revista Iberoamericana de Evaluación Educativa, 5(1), 318-327. http://www.rinace.net/riee/numeros/vol5num1_e/art24.pdf

Tejedor, F. J. \& Jornet, J. M. (2008). La evaluación del profesorado universitario en España. Revista Electrónica de Investigación Educativa, 10(SPE.), 1-29. 
http://redie.uabc.mx/index.php/redie/article/v $\underline{\text { iew/199/339 }}$

Theall, M. \& Feldman, K.A. (2007). Commentary and update on Feldman's (1997) "Identifying exemplary teachers and teaching: Evidence from student ratings". En R.P. Perry \& J.C. Smart (eds.), The Scholasship of Teaching and Learning in
Higher Education: An Evidence-Based Perspective, (pp.130-143). Netherlands: Springer.

Unidad Técnica de Evaluación y Mejora de la Calidad Docente (2003). Informe general sobre la calidad docente del profesorado de la ULL. La Laguna: Servicio de Publicaciones de la Universidad.

\section{Autor}

To know more / Saber más

Casero Martínez, Antonio (a.casero@uib.es).

Licenciado en Psicología y Doctor en Ciencias de la Educación. Profesor Contratado Doctor del Área de conocimiento de Didáctica y Organización

Escolar de la Universitat de les Illes Balears. Miembro del grupo de investigación Educación y Ciudadanía (EIC). La actividad investigadora se centra, principalmente, en el ámbito de la calidad de la docencia universitaria. Su dirección postal es: Departamento de Pedagogía Aplicada y Psicología de la Educación, edificio Guillem Cifre de Colonya, Crta. Valldemossa, km, 7.5. 07122 - Palma (España).

\section{RELIEVE}

\section{Revista ELectrónica de Investigación y $\mathbf{E V}$ aluación Educativa E-Journal of Educational Research, Assessment and Evaluation}

[ISSN: 1134-4032]

(C) Copyright, RELIEVE. Reproduction and distribution of this articles it is authorized if the content is no modified and their origin is indicated (RELIEVE Journal, volume, number and electronic address of the document).

(C) Copyright, RELIEVE. Se autoriza la reproducción y distribución de este artículo siempre que no se modifique el contenido y se indique su origen (RELIEVE, volumen, número y dirección electrónica del documento). 